\title{
L'Accroissement de Risque: Théorie et Application à la Production en Incertitude
}

\author{
par Louis Eeckhoudt*
}

\section{Introduction}

Alors que la notion de risque est maintenant formalisée de façon claire et largement admise, il est surprenant de constater qu'il existe plusieurs definitions concurrentes d'un "accroissement de risque".

L'objet du présent article consiste à comparer les définitions existantes ${ }^{1}$ et à en proposer une nouvelle, tout en évaluant leurs mérites respectifs. Comme l'indique le titre de l'article, ces définitions sont formulées dans un cadre particulier: celui de la décision de production en incertitude de prix. Cette application a été choisie non seulement pour rendre l'exposé moins abstrait, mais aussi parce qu'une "bonne" définition doit pouvoir être appliquée avec fruit dans des questions concrètes.

Dans la section 2 de l'article, le modèle de production en incertitude de prix est décrit par référence à deux articles importants sur le sujet, ceux de Baron [1] et de Sandmo [7]. Dans la section 3, nous présentons quatre définitions existantes de la variation de risque et nous examinons leurs implications pour le problème décrit en 2 . Leurs insuffisances motivent la nouvelle définition présentée dans la section 4 . Enfin, nous avons cru utile, en conclusion (section 5), de montrer les liens entre notre définition et le problème fondamental de l'assurance.

\section{La production en incertitude de prix}

Nous nous référons ici au cas le plus simple, celui de la concurrence parfaite, dans lequel la firme n'a aucune action possible sur les prix ${ }^{2}$. Il est supposé également que les coûts de production sont connus avec certitude. Par contre, le prix que la firme recevra plus tard est incertain et nous convenons donc de le représenter par une variable aléatoire. Suivant Sandmo, la notation suivante est utilisée:

* Faculté Universitaire Catholique de Mons (Belgique). Cet article doit beaucoup à ma coopération avec mes collègues $\mathrm{Ph}$. Capéraà et $\mathrm{P}$. Hansen.

1 Il faudrait dire "certaines définitions existantes", car la littérature en contient plus que celles recensées ici.

2 Plusieurs articles ont été écrits sur la concurrence imparfaite. Voir, par exemple, Leland [5]. Toutefois, pour simplifier l'exposé, nous n'y ferons pas allusion. 
$\mathrm{x} \quad$ niveau de production

$\mathrm{c}(\mathrm{x})$ coût variable total avec c'(x), le coût marginal positif

$\widetilde{p} \quad$ le prix aléatoire avec une densité de probabilité $f(p)$ et une espérance mathématique $\mathrm{E}(\widetilde{\mathrm{p}})$

B le coût fixe

Dès lors, le profit s'écrit :

$\widetilde{\pi}=\widetilde{\mathrm{px}}-\mathrm{c}(\mathrm{x})-\mathrm{B}$

et si la firme a comme objectif la maximisation de son utilité espérée (E[U]), la valeur optimale de $\mathrm{x}\left(\mathrm{x}^{*}\right)$ doit satisfaire:

(2.1) $\mathrm{E}\left[\mathrm{U}^{\prime} \cdot\left(\mathrm{p}-\mathrm{c}^{\prime}\left(\mathrm{x}^{*}\right)\right)\right]=0$

A partir de ce résultat, Sandmo démontre que la valeur retenue pour x en incertitude $\left(x^{*}\right)$ est inférieure à celle que choisirait la firme si elle était confrontée à un prix certain égal à $\mathrm{E}(\widetilde{\mathrm{p}})$. Ce résultat est obtenu à condition que la firme éprouve une aversion pour le risque.

\section{Quatre définitions de l'accroissement de risque}

3.1 La première définition analysée ici est celle de Sandmo, proposée dans son article de 1971. Cette définition a le mérite de bien correspondre a notre intuition. On dit que la nouvelle variable aléatoire $\widetilde{p}$ ' est plus risquée que $\widetilde{p}$ si elle conserve la même espérance tout en ayant une densité plus "étirée" 3 . En termes graphiques, on obtient la figure 1:

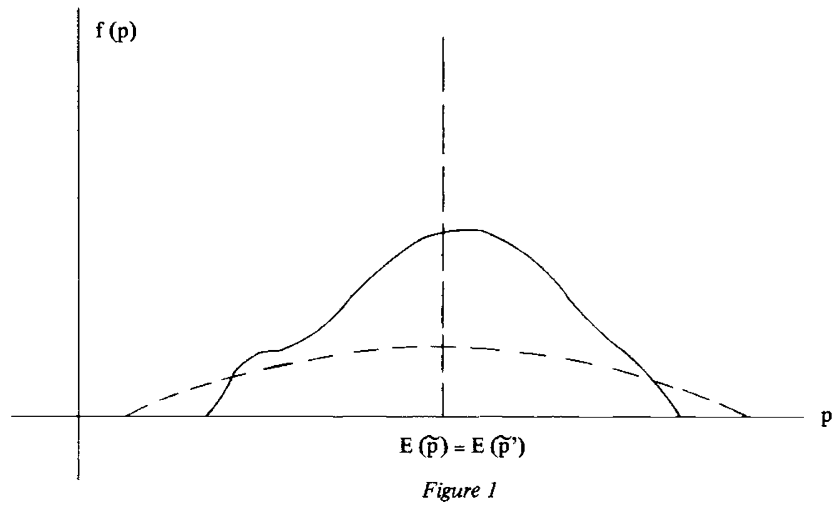

La densité initiale est en traits continus et la nouvelle, en traits discontinus. Bien entendu, la densité représentée en discontinu est plus risquée que la précédente.

\footnotetext{
${ }^{3}$ Sandmo parle d'un "stretching" de la densité de prix.
} 
En termes plus rigoureux, on définit $\widetilde{\mathrm{p}}$ ' comme suit:

$$
\begin{aligned}
& \widetilde{p}^{\prime}=\gamma \widetilde{p}+S \\
& \text { où } \gamma \text { et } S \text { sont des constantes }
\end{aligned}
$$

Si on part de $\gamma=1$ et $S=0$ et si on accroît légèrement $\gamma$ avec $S$ qui varie pour conserver $\mathrm{E}$ constant, on dit que $\widetilde{\mathrm{p}}$ ' devient plus risqué que $\widetilde{\mathrm{p}}$.

A l'aide de cette définition, Sandmo démontre que l'accroissement de risque entraîne une réduction de la production quand l'aversion absolue pour le risque (Aa) est une fonction décroissante de la richesse ${ }^{4}$. Ce résultat mérite quelque commentaire. Bien sûr, intuitivement, nous pensons que l'accroissement de risque décourage la production mais, pour obtenir ce résultat, Sandmo a besoin d'une condition restrictive: la décroissance de Aa. Cette remarque motivera dans la section suivante notre recherche d'une nouvelle définition de la variation de risque.

3.2 Dès 1971, Rotschild et Stiglitz [6] ont présenté trois autres définitions de la variation de risque. Nous en présentons ici deux en détail et nous citons la troisième. Ensuite, nous indiquons leur inter-relation.

A. A nouveau, de façon intuitive, on peut dire que $\widetilde{p}$ ' est plus risqué que $\widetilde{p}$ si $\widetilde{p}$ ' est obtenue à partir de $\widetilde{\mathrm{p}}$ en prenant du "poids" au milieu de la densité de $\widetilde{\mathrm{p}}$ et en plaçant ce poids dans les queues de la distribution. Cette opération sur $\widetilde{p}$ est effectuée de manière telle que l'espérance de $\widetilde{p}$ reste constante.

En termes graphiques, on obtient:

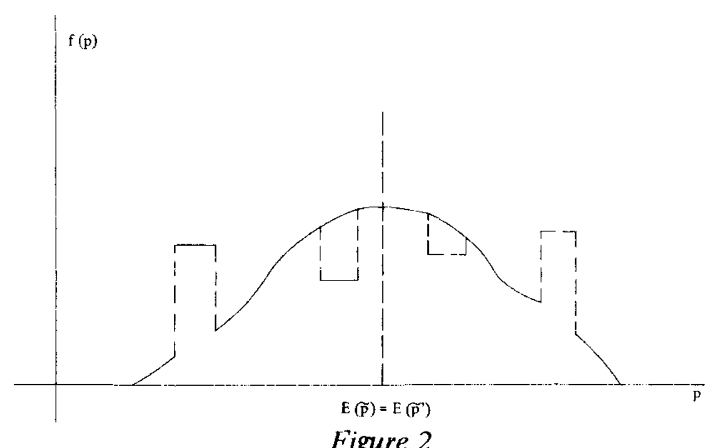

Figure 2

A nouveau, la densité plus risquée est en traits discontinus.

4 Pour être complet, il faut signaler que cette démonstration a été faite par Coes [2] et Ishii [4] beaucoup plus récemment à partir de l'article de Sandmo paru en 1971. Nous devons aussi ajouter que la décroissance de $A_{a}$ est une condition suffisante mais non nécessaire pour obtenir le résultat. 
B. Une autre façon de définir l'accroissement de risque consiste à dire que $\widetilde{\mathrm{p}}$ ' est obtenue à partir de la variable aléatoire $\widetilde{\mathrm{p}}$ à laquelle a été ajoutée un bruit $\widetilde{\mathrm{X}}$ d'espérance nulle pour toute réalisation $\mathrm{p}$ de $\widetilde{\mathrm{p}}$, c'est-à-dire :

(3.2) $\mathrm{E}(\widetilde{\mathrm{X}} / \widetilde{\mathrm{p}}=\mathrm{p})=0$

de sorte que $E\left(\widetilde{p}^{\prime}\right)=E(\widetilde{p})$.

Nous illustrons cette définition pour le cas où $\widetilde{p}$ suit une loi discrète:

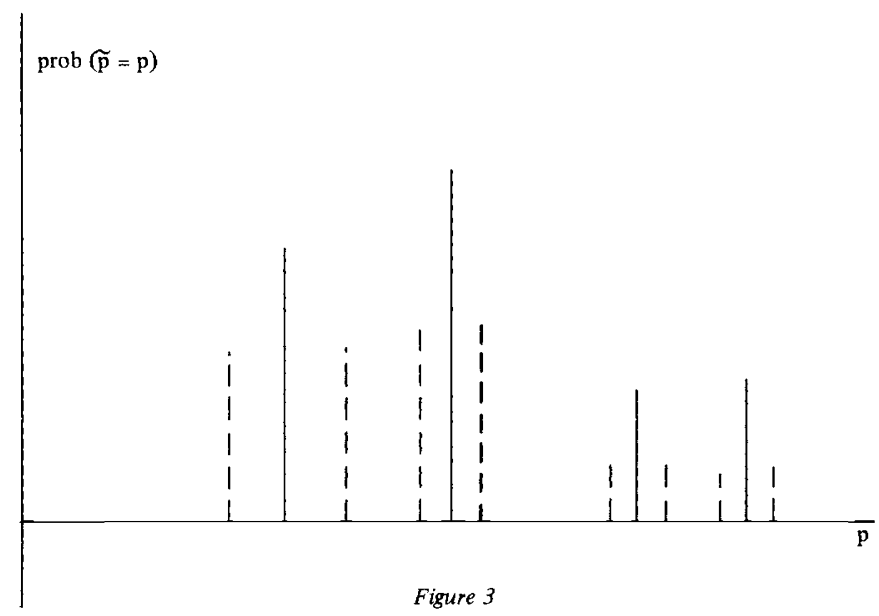

A la figure 3, nous avons maintenu notre convention de représenter la variable aléatoire plus risquée en discontinu et nous avons supposé que ce bruit $\mathrm{X}$ pouvait prendre 2 valeurs, une positive et une négative avec égale probabilité.

C. Enfin, la troisième définition de Rotschild et Stiglitz est seulement évoquée ici: on dit que $\widetilde{\mathrm{p}}$ ' est plus risquée que $\widetilde{\mathrm{p}}$ si toutes les personnes qui ont une aversion pour le risque préfèrent la distribution de $\widetilde{\mathrm{p}}$ à celle de $\widetilde{\mathrm{p}}$ '.

Une des contributions essentielles de Rotschild et Stiglitz consiste à établir que ces trois définitions apparemment très différentes conduisent au même classement des variables aléatoires et donc qu'elles sont, en fait, équivalentes. De plus, ils montrent que la définition de Sandmo, en dépit de son intérêt intuitif, n'est pas comparable aux trois autres.

On peut, dès lors, dire que les définitions de Rotschild et Stiglitz présentent un progrès en termes de logique par rapport à celle de Sandmo. Néanmoins, au même titre que cette dernière, leur application à des problèmes de décision exige des hypothèses très restrictives sur la fonction d'utilité pour obtenir des résultats non ambigus.

Cette faiblesse motive l'approche présentée dans la section 4 . 


\section{La variation de risque définie à partir des systèmes de prix minimum et maximum}

La notion de risque étant, dans une large mesure, subjective, il n'est pas étonnant que les individus revoient leurs estimations relatives au futur et que des changements du risque, tel qu'il est perçu par chaque agent économique, se produisent de manière fréquente. Néanmoins, il est difficile d'imaginer des chocs exogènes qui introduiraient précisément des variations de risque exactement identiques à celles définies en 3.

Par contre, sur certains marchés - et celui des changes est un bel exemple - des interventions extérieures sous forme de prix minimum et maximum entraînent des variations de risque. C'est pourquoi dans cette section, nous étudions séparément l'effet des prix minimum et maximum sur les décisions de production avant de les combiner pour présenter notre définition de l'accroissement de risque.

Si un gouvernement ou une quelconque agence garantit à un producteur un prix minimum, celui-ci revoit ses attentes de prix de la façon décrite à la figure 4 .

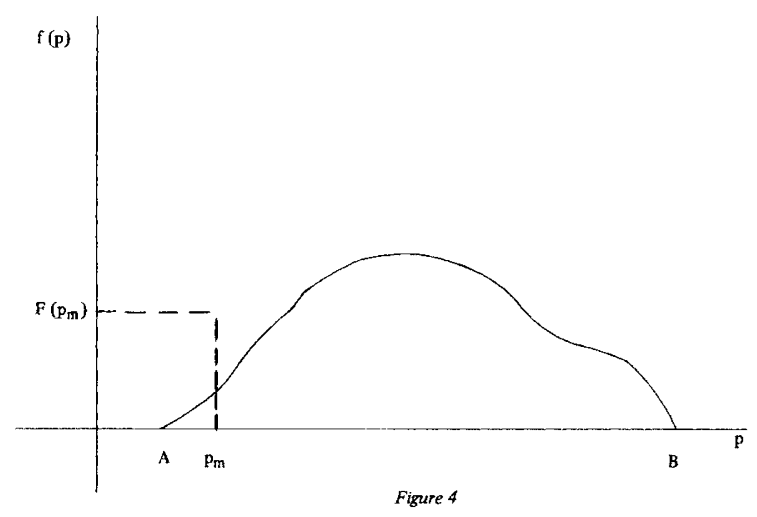

La densité initiale prenait des valeurs non nulles dans l'intervalle [A, B]. Si on lui propose un prix minimum $\left(\mathrm{p}_{\mathrm{m}}\right)$, il est maintenant sûr de ne plus observer de prix inférieur à $\mathrm{p}_{\mathrm{m}}$, de sorte que, dans cet intervalle, $\mathrm{f}(\mathrm{p})=0$. A $\mathrm{p}=\mathrm{p}_{\mathrm{m}}$, il attache maintenant une probabilité $F\left(p_{m}\right)=\int_{m}^{p_{m}} f(p) d p$ et pour les prix supérieurs à $p_{m}$, la nouvelle densité A

coïncide avec l'ancienne.

Il a été montré en [3] que l'introduction de ce prix minimum ou son accroissement stimulent la production quand la firme éprouve une aversion pour le risque.

On aurait pu croire a priori que l'augmentation d'un prix maximum $\left(\mathrm{p}_{M}\right)$ autorisé devait conduire au même résultat. En fait, ce n'est pas nécessairement vrai parce que, 
comme on peut le démontrer, l'augmentation de $\mathrm{p}_{\mathrm{M}}$ accroît à la fois l'espérance et la

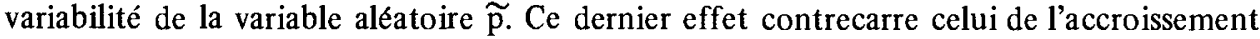
de l'espérance pour un producteur qui craint le risque et, dès lors, dans certains cas, l'augmentation de $\mathrm{p}_{\mathrm{M}}$ entraîne une réduction de production.

Bien entendu, des changements séparés de $\mathrm{p}_{m}$ ou de $\mathrm{p}_{\mathrm{M}}$ ne conduisent pas à des variations de risque telles qu'elles ont été définies en 3 puisque, dans chacun des cas, l'espérance de $\widetilde{\mathrm{p}}$ se trouve modifiée. Pour que celle-ci reste constante, il faut considérer des valeurs de $\mathrm{p}_{m}$ et de $\mathrm{p}_{\mathrm{M}}$ telles que la relation suivante soit respectée:

$$
\int_{A}^{B} p f(p) d p=p_{m} F\left(p_{m}\right)+\int_{p_{m}}^{p_{M}} p f(p) d p+p_{M}\left(1-F\left(p_{M}\right)\right)
$$

En différenciant totalement (4.1), on trouve pour une valeur constante de $E(\widetilde{p})$ : (4.2) $\frac{d p_{M}}{d p_{m}}=\frac{F\left(p_{m}\right)}{1-F\left(p_{M}\right)}$

A partir de ce résultat, il a été montré en [3] qu'une diminution (augmentation) de risque, définie à partir de $p_{m}$ et de $p_{M}$, conduit nécessairement à une augmentation (diminution) de la production si la firme a de l'aversion pour le risque.

Ce résultat ne manque pas d'intérêt. En premier lieu, une affirmation souvent rencontrée ("les accroissements de risque découragent l'investissement") se trouve confirmée par un modèle théorique à partir d'une hypothèse largement admise: l'existence de l'aversion pour le risque. Il est important de rappeler que les autres définitions de l'accroissement de risque ne donnent le résultat attendu que moyennant des hypothèses restrictives sur la forme de la fonction d'utilité. En outre, la proposition énoncée cidessus permet de retrouver le résultat de Sandmo rappelé en 2 comme corollaire de la formule (2.1). En effet, si on continue à augmenter $p_{m}$ et à diminuer $p_{M}$ tout en laissant $E(\widetilde{p})$ constant on finit par trouver $p_{m}=p_{M}=E(\widetilde{p})$. A ce moment, toute l'incertitude a disparu et on trouve conformément à notre proposition que la production correspondant à $\mathrm{p}_{\mathrm{m}}=\mathrm{p}_{\mathrm{M}}=\mathrm{E}(\widetilde{\mathrm{p}})$ est plus grande que celle obtenue auparavant. Ceci n'est rien d'autre que le résultat de Sandmo apparaissant ici comme le cas limite de la compression progressive de la distribution de $\widetilde{p}$.

\section{Relations avec l'assurance}

Si la notion de prix minimum et maximum permet d'introduire une définition intéressante des variations de risque, elle n'est pas non plus sans relation avec les problèmes d'assurance. En effet, lorsque un gouvernement propose à une entreprise un système de prix tel que celui décrit en 4 , ii rédige en fait une police d'assurance un peu particulière. L'objet de l'assurance est clair: la firme est protégée complètement contre la survenance d'un événement "négatif" pour elle, à savoir la perception d'un prix inférieur à $\mathrm{p}_{\mathrm{m}}$. Par contre, la prime qu'elle paie est aussi aléatoire: il s'agit du sacrifice qu'elle fait en ne percevant pas un prix supérieur à $\mathrm{p}_{\mathrm{M}}$. Enfin, le contrat est neutre sur le plan actuariel parce que $p_{m}$ et $p_{M}$ sont choisis de manière telle que $E(\widetilde{p})$ reste constant. 
Dès lors, on peut se poser la question suivante: n'existerait-il pas des formes d'assurance autres que celle des prix minimum et maximum qui, tout en protégeant aussi bien la firme, seraient moins coûteuses pour le gouvernement? Malheureusement, la réponse à cette question importante requiert des outils d'analyse extrêmement élaborés. Quoi qu'il en soit, il s'agit sans doute d'un champ de recherches fort intéressant et qui mérite l'attention des économistes de l'assurance.

\section{REFERENCES}

1. BARON, D.: "Price uncertainty, utility, and industry equilibrium in pure competition", International Economic Review, 11 (octobre 1970), 463-480.

2. COES, D.: "Firm out put and change in uncertainty", American Economic Review, 67 (mars 1977), 249-251.

3. EECKHOUDT, L. et P. HANSEN: "Minimum and maximum prices, uncertainty and the theory of the competitive firm", à paraître in American Economic Review.

4. ISHII, Y.: "On the theory of the competitive firm under price uncertainty: Note", American Economic Review, 67 (septembre 1977), 768-769.

5. LELAND, H.: "Theory of the firm facing uncertain demand", American Economic Review, 62 (juin 1972), 278-291.

6. ROTHSCHILD, M. et J. STIGLITZ: "Increasing risk I: A definition", Journal of Economic Theory, 2 (septembre 1970), 225-243.

7. SANDMO, A.: "On the theory of the competitive firm under price uncertainty", American Economic Review, 61 (mars 1971), 65-73. 